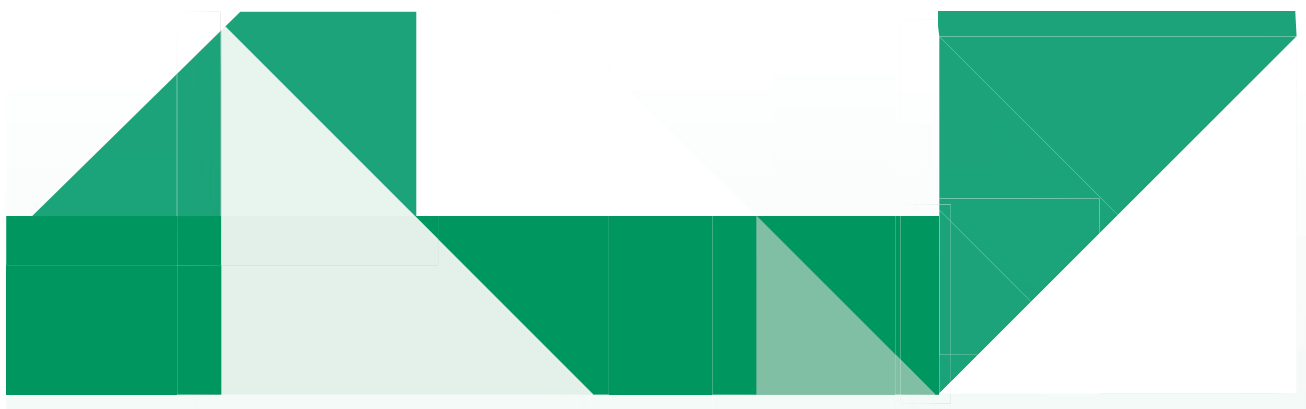

\title{
D) El problema de la teleología y la metáfora del diseño en biología: cuestiones epistemológicas e implicancias didácticas
}

- O problema da teleologia e a metáfora do disenho em biologia: questãos epistemologicas e implicaçãos didaticas

- The Problem of Teleology and the Metaphor of Design in Biology: Epistemolgical Issues and Didactic Implications

\section{Resumen}

La existencia y resistencia de muchas concepciones alternativas de los estudiantes se reconoce como uno de los principales problemas en la enseñanza de la teoría de la evolución. Estas concepciones suponen frecuentemente razonamientos teleológicos. De acuerdo con la perspectiva más difundida sobre este problema, la teleología no ocupa un lugar legítimo en la biología y, sobre esta base, se propone como objetivo didáctico la modificación y el reemplazo, es decir, la eliminación de las concepciones teleológicas de los estudiantes. Este ha sido el supuesto básico sobre el que se han elaborado y probado numerosas propuestas de aprendizaje por cambio conceptual cuyos resultados han sido más bien limitados. En este trabajo cuestionamos las bases epistemológicas de esta "perspectiva tradicional", proponemos un enfoque alternativo y ofrecemos algunos lineamientos para el tratamiento didáctico de este problema. Sugerimos que el lenguaje teleológico en la biología puede entenderse como una expresión de la metáfora del diseño. Dicha metáfora es adecuada porque los seres vivos parecen diseñados en virtud de su carácter adaptativo que, a su vez, se explica por ser producto de la selección natural. Sostenemos, además, que la explicación darwiniana de la adaptación es teleológica, entre otras razones porque supone apelar necesariamente a la metáfora del diseño. Discutimos el rol de las metáforas y analogías en la enseñanza de la ciencia y en el desarrollo de la ciencia misma para concluir que las metáforas son parte de la creación científica y de sus productos, las teorías científicas. Sobre estas bases sugerimos un trabajo didáctico centrado en el análisis explícito de la metáfora del diseño por parte de los estudiantes. En esta propuesta el foco está puesto en el desarrollo de las capacidades metacognitivas de los estudiantes, de modo
Leonardo González Galli*

Dr. en Ciencias Biológicas y Profesor de Enseñanza Media y Superior en Biología por la Universidad de Buenos Aires. Se desempeña como investigador asistente del Consejo Nacional de Investigaciones Científicas y Tecnológicas (Conicet, Argentina) y como profesor adjunto en el Profesorado de Biología de la Facultad de Ciencias Exactas y Naturales de la Universidad de Buenos Aires (Argentina). Dirige y orienta clases en la Escuela Argentina de Naturalistas (perteneciente a la ong ambientalista Aves Argentinas / Asociación Ornitológica del Plata). ORCID: orcid.org/0000-0002-0713-164X 
que comprendan en qué consiste la metáfora del diseño, puedan reconocerla y puedan regular su uso. Creemos que dado el estatus problemático de la teleología en la biología y el consenso sobre el rol constitutivo y funcional en la cognición humana de este tipo de razonamientos el objetivo de que los estudiantes los abandonen no es realista ni deseable. El enfoque propuesto tiene otras virtudes, tales como aportar a la construcción por parte de los estudiantes de una imagen de ciencia más adecuada a partir del aprendizaje en relación con el rol de las metáforas en la ciencia.

Palabras clave

Evolución, teleología, metáfora, enseñanza

\section{Resumo}

A existência e resistência de muitas concepções alternativas dos alunos se reconhecem como um dos principais problemas no ensino da teoria da evolução. Estas concepções supõem com frequência argumentações teleológicas. De acordo com a perspectiva mais difundida acerca deste problema, a teleologia não ocupa um lugar legitimo na biologia e, sobre esta base, se propõe, como objetivo didático a modificação das concepções teleológicas dos alunos. Isto foi o suposto básico sobre o qual muitas propostas de aprendizagem por mudança conceitual foram feitas; os resultados foram bastante limitados. Neste trabalho questionamos as bases epistemológicas desta "perspectiva tradicional", propomos uma abordagem alternativa e oferecemos algumas orientações para o tratamento didático de este problema. Sugerimos que a linguagem teleológica na biologia pode ser entendida como uma expressão da metáfora do design. Essa metáfora é apropriada porque os seres vivos parecem concebidos com caráter adaptativo, o que, por sua vez, é consequência de ser um produto da seleção natural. Afirmamos, alem disso, que a explicação darwiniana da adaptação é teleológica, entre outras razoes, porque necessariamente deve apelar para a metáfora do design. Discutimos o papel das metáforas e analogias no ensino da ciência e no desenvolvimento da propia ciência, para concluir que as metáforas fazem parte da criação científica e também dos seus produtos, as teorias científicas. Sobre estas bases sugerimos um trabalho didático focado na análise explicito da metáfora do design pelos alunos. Nesta proposta, o foco está no desenvolvimento das habilidades metacognitivas dos alunos para que eles entendam o que é a metáfora do design, conseguindo reconhocer-la e regulando a sua utilização. Acreditamos que, dado o estatus problemático da teleologia na biologia e o consenso sobre o papel constitutivo e funcional na cognição humana deste tipo de raciocínios, não é realista nem desejável que sejam abandonados pelos alunos. A abordagem apresentada tem outras virtudes, tais como contribuir para a construção pelos alunos de uma imagem de ciência mais adequada partindo do aprendizagem relativo ao papel das metáforas na ciência.

Palavras chave

Evolução, teleologia, metáfora, ensino

\section{Abstract}

The existence and resistance of many students' alternative conceptions is regarded as one of the main problems in the teaching of the theory of evolution. These conceptions frequently involve teleological reasoning. The most widely spread perspective on this problem states that teleology does not play a legitimate role in biology. Based upon this assumption, it proposes as a didactic aim the modification and replacement- - that is to say, the elimination —of students' teleological conceptions. Learning by conceptual change proposals have been built and tested upon this assumption, with rather limited results. In this paper, we question the epistemological bases of this "traditional perspective", we propose an alternative approach and provide some guidelines to address this issue didactically. We also suggest that the teleological language in biology may be understood as an expression of the design metaphor. Such metaphor is suitable because organisms seem to be designed by virtue of their adaptive character, which, in turn, is a consequence of being a product of natural selection. Furthermore, we assure that Darwin's explanation of adaptation is teleologi- 
cal, among other reasons, because it necessarily implies to appeal to the metaphor of design. We discuss the role of metaphors and analogies in the teaching of science and in the development of science itself, in order to infer that metaphors are part of scientific creation and its products, that is, scientific theories. Consequently, we suggest a didactic work focused in the explicit analysis of the metaphor of design by students. In this proposal, the focus is on developing students' metacognitive skills, so that they can understand what the metaphor of design is, recognize it and regulate its use. We think that because of the problematic status of teleology in biology and the consensus about the constitutive and functional role played by this kind of reasoning in human cognition, it is neither realist nor desirable that students would give them up. The approach proposed has other virtues, such as contributing to students' construction of a more appropriate image of science, as a consequence of learning in connection with the role of metaphors in science.

Keywords

Evolution, teleology, metaphor, teaching 


\section{Introducción}

La teoría de la evolución (TE) ocupa un lugar central en la biología contemporánea (Futuyma, 2009). Aunque es difícil evaluar la importancia relativa de cada uno de los modelos que integran la TE, el modelo de evolución por selección natural (MESN) ocupa, sin duda, un lugar destacado (Dupré, 2006; Futuyma, 2009). La centralidad del MESN se puede defender señalando que ofrece una primera respuesta a los dos grandes interrogantes de la biología evolutiva: el origen de la diversidad biológica (la selección natural es parte de la mayoría de los modelos de especiación) y el origen de la adaptación. En relación con el segundo problema (adaptación), el MESN constituye, de hecho, la única explicación científicamente aceptada del "ajuste" observado entre los organismos y sus circunstancias ambientales (Dennett, 1995; Sterelny y Griffiths, 1999). Por otro lado, la TE tiene diversas y profundas implicancias que van más allá del dominio de la biología (Stamos, 2009). Estos breves señalamientos alcanzan para justificar un lugar central para la TE en general, y especialmente para el MESN, en el currículo de la enseñanza general y obligatoria en ciencias. Sin embargo, existe acuerdo tanto sobre este punto como sobre el hecho de que, en general, los resultados de la enseñanza en relación con este contenido son extremadamente bajos (Smith, 2010). Numerosas investigaciones llevadas a cabo en diversos países desde hace ya más de tres décadas muestran que el público general egresa del sistema educativo básico sin haber aprendido los principios elementales de la biología evolutiva (Smith, 2010).

Se han postulado diversas causas de esta situación, entre las cuales se destaca la existencia y resistencia al cambio de un conjunto de concepciones alternativas. Un rasgo central de estas concepciones es que suelen suponer razonamientos finalistas o teleológicos (véanse, por ejemplo, Bishop y Anderson, 1990; Hallden, 1988 y Settlage, 1994). Casi sin excepción, el carácter teleológico de estas concepciones ha sido considerado un rasgo erróneo y negativo. Esta postura se ha adoptado a partir de asumir que los razonamientos teleológicos no cumplen ningún rol legítimo en la biología "erudita" y ha derivado en diversas propuestas de "cambio conceptual" dirigidas a erradicar la teleología de los razonamientos de los estudiantes. La mayoría de los biólogos (por ejemplo, Futuyma, 2009), profesores de biología e investigadores en didáctica de las ciencias han adoptado esta postura. Como excepciones, debemos mencionar a Jungwirth (1975a, 1975b), quien señaló que la teleología no implica necesariamente intencionalidad ni supuestos sobrenaturales y denunció, correctamente según creemos, como "dogmática" la actitud imperante sobre este problema en el ámbito de la educación. Sin embargo, este autor concluye su artículo señalando que el objetivo último sería "... la gradual eliminación del antropomorfismo de los estudiantes" ${ }^{\prime}$. 
Como otra excepción, Zohar y Ginossar (1998) y Tamir y Zohar (1991) han valorado positivamente el potencial heurístico del pensamiento teleológico. Los primeros invitan -en lo que denominan "sugerencias heréticas" - a flexibilizar la censura dogmática de la teleología en la enseñanza de la biología teniendo en cuenta tres argumentos: (1) los intentos de censura fracasan en la práctica, (2) los razonamientos teleológicos tienen valor heurístico y (3) entre los filósofos de la biología no existe un consenso sobre la legitimidad de la teleología.

En síntesis, la TE en general, y el MESN en particular, constituyen un contenido central en la enseñanza de la biología en la educación general obligatoria. Por otro lado, los logros en términos de aprendizaje son, en general, deficientes. La existencia de concepciones alternativas altamente resistentes al cambio parece ser uno de los principales factores que condicionan el aprendizaje de estos contenidos y un rasgo notorio de estas concepciones es su carácter teleológico. Suponiendo que la teleología no ocupa un lugar legítimo en la biología contemporánea, y que es contradictoria con el MESN, la mayoría de los investigadores y educadores han propuesto como uno de los objetivos de la enseñanza eliminar y reemplazar las nociones teleológicas. En muchos casos, esto se ha traducido en una censura del lenguaje teleológico en las clases (Zohar y Ginossar, 1998). Llamaremos a este conjunto de supuestos la "perspectiva tradicional" sobre el problema de la teleología en la enseñanza.

En consonancia con las críticas de Zohar y Ginossar (1998), Tamir y Zohar (1991) y Jungwirth (1975a, 1975b), pero yendo más allá de ellas y en contra de la "perspectiva tradicional", sugerimos que las nociones teleológicas cumplen un rol preeminente y legítimo en la biología actual y, más específicamente, en el
MESN. Sostenemos además que la teleología forma parte de la biología en virtud de que los seres vivos parecen diseñados como consecuencia de que son producto de la selección natural y, siguiendo a Ruse (2000), que el MESN, que permite explicar dicho "diseño biológico", supone un recurso obligado a la metáfora del diseño (MD). Esta metáfora sugiere que la selección natural es como un artífice humano o, lo que es lo mismo, que los organismos, o sus partes, son como artefactos. Esta propuesta no pretende ser una solución a los arduos problemas epistemológicos implicados sino, más bien, una toma de posición en relación con dichos problemas que resulte útil a los fines didácticos.

Esta perspectiva epistemológica, basada principalmente en los análisis de Michael Ruse (2003, 2000), no supone que las intuiciones teleológicas de los estudiantes sean "científicamente correctas". De hecho, en otros trabajos hemos caracterizado la "teleología de sentido común" de los estudiantes como un obstáculo para el aprendizaje del MESN (González Galli y Meinardi, 2015; 2011 ). El concepto didáctico de obstáculo fue propuesto y desarrollado principalmente por didactas de las ciencias naturales franceses (Astolfi y Develay, 1989) y está "inspirado" en el concepto de "obstáculo epistemológico" del filósofo Gaston Bachelard (2004).

Este autor entendía por "obstáculo" un sistema de pensamiento instalado en la comunidad científica que impide la construcción de nuevos modos de ver los problemas, obturando, de este modo, el progreso científico. Aunque el mismo Bachelard exploró la analogía entre este aspecto de la historia de la ciencia y el aprendizaje individual fueron -como dijimos-, otros autores quienes desarrollaron la "versión didáctica" del concepto. Tal vez, la principal diferencia entre el concepto bachelardiano original y el didáctico es que el 
primero tiene un carácter totalmente negativo mientras que desde la perspectiva didáctica se reconoce que el obstáculo tiene a la vez un efecto posibilitador y limitante del aprendizaje (Astolfi, 1999). Siguiendo a Astolfi hemos propuesto (González Galli y Meinardi, 2010) una definición de acuerdo con la cual un obstáculo es una concepción transversal (tiene cierto grado de generalidad), funcional (le sirve al sujeto para dar cuenta de ciertos fenómenos) y conflictiva (en cierto sentido, "compite" con el modelo científico que pretendemos enseñar como marco teórico de referencia). Así, caracterizado como un obstáculo, el pensamiento teleológico sigue siendo un problema central para la enseñanza de la TE (González Galli y Meinardi, 2011 ). Lo que estamos cuestionando es la "perspectiva tradicional" en relación con dicho problema. En este sentido, y desde el marco teórico de los "obstáculos epistemológicos" (Peterfalvi, 2001), en los mencionados trabajos hemos sugerido que el principal objetivo didáctico no sería la eliminación del pensamiento teleológico sino el desarrollo de una "vigilancia" metacognitiva por parte de los estudiantes. Esto supondría la capacidad de identificar los razonamientos teleológicos y de juzgar en qué casos son legítimos y en cuáles no lo son. En este trabajo precisaremos un modo de entender el rol de la teleología en la biología y, a partir de ese análisis, sugeriremos algunas líneas de acción para el desarrollo y la implementación de estrategias didácticas dirigidas a mejorar el aprendizaje de la TE a partir del trabajo sobre las concepciones teleológicas de los estudiantes.

\section{Las explicaciones teleológicas}

No existe una única definición de explicación teleológica. Algunos autores solo consideran como tales aquellas explicaciones que incluyen un componente intencional (Estany, 1993) mientras que otros incluyen en esta categoría cualquier explicación que haga referencia a las nociones de meta, fin u objetivo así como a conceptos relacionados, como los de diseño y función² (Gaeta, Gentile, Lucero y Robles, 1996). En este trabajo adoptaremos la segunda perspectiva. Así, si nos preguntamos "¿́por qué las flores de una especie producen perfume?", la respuesta "producen perfume para atraer a los polinizadores" sería teleológica aunque no supusiera ninguna forma de intencionalidad.

El rechazo a las explicaciones teleológicas, que ganó fuerza a partir de la Revolución Científica, se funda en tres argumentos. El primero se refiere a la asociación de estas explicaciones con sistemas de pensamiento que suponen la existencia de agentes sobrenaturales (Allen et al., 1998; Gaeta et al. 1996). El segundo se basa en que estas explicaciones no parecen ajustarse al modelo nomológico deductivo de explicación científica (Díez y Moulines, 1999; Gaeta et al., 1996). Finalmente, las explicaciones teleológicas serían contradictorias con la noción clásica de causalidad debido a que parecen invertir la relación

2 Sobre el problemático concepto de función en la biología véanse Chediak (2011, 2006), Nunes-Neto et ál. (2013) y Nunes-Neto y El-Hani (2009). 
temporal entre la causa y el efecto al suponer que el fin (temporalmente posterior) causa, de algún modo, el fenómeno que se intenta explicar (temporalmente anterior) (Díez y Moulines, 1999; Estany, 1993). El único ámbito en el que, en principio, este tipo de explicaciones no resulta conflictivo es el de las acciones humanas voluntarias y conscientes. Aunque suele asociarse a otras nociones, como el antropomorfismo, la intencionalidad y el vitalismo, la teleología, entendida como cualquier apelación a la noción de fin o equivalente, no supone necesariamente ninguna de estas otras ideas (Jungwirth, 1975a; Nagel, 2006).

\section{La teleología en la biología}

Las explicaciones teleológicas han tenido una importancia variable en los sistemas conceptuales de los diferentes filósofos y científicos a lo largo de la historia (para un análisis histórico de esta cuestión, véase Ruse, 2003). En el ámbito de la biología evolutiva, el lugar de la teleología en los distintos sistemas conceptuales puede relacionarse con un gran debate que, según Gould (2004), se expresa en la oposición entre estructuralismo y funcionalismo, orden y teleología, morfología y adaptación o unidad de tipo y condiciones de existencia. Los primeros miembros de cada par expresan una visión del mundo menos propensa a las explicaciones teleológicas que los segundos. Por ejemplo, en el primer grupo encontramos autores como Louis Agassiz y Étienne Geoffroy Saint-Hilaire y en el segundo a Georges Cuvier y Charles Darwin. Contemporáneamente, podríamos agregar al mismo Stephen Gould a la primera lista y a Richard Dawkins a la segunda. Lo importante en relación con el objetivo de este trabajo es que, de acuerdo con la versión más difundida de la historia, Charles Darwin habría ofrecido una explicación puramente naturalista del "diseño biológico" (adaptación) y con ello habría eliminado la teleología de la biología. Aunque nadie duda de lo primero, lo segundo no es tan claro (Allen, 2003). En efecto, para algunos autores (Ruse, 2003; Sober, 1996), lejos de eliminar la teleología, el darwinismo la naturalizó y, con ello, la legitimó.

Más allá de este debate hay un hecho innegable: Darwin utilizó profusamente el lenguaje teleológico (Lennox, 1993) y los biólogos, desde entonces, nunca han dejado de hacerlo (Rosenberg y McShea, 2008; Ruse, 2003). Aunque es posible encontrar expresiones teleológicas en otras ciencias, sin duda estas son más frecuentes en biología (Lewens, 2004; Mahner y Bunge, 2000). Por ejemplo, en un libro de bioquímicia leemos que "Los coágulos [...] están diseñados para disolverse cuando se restaura la integridad estructural de las zonas dañadas." (Stryer, 1995). La cuestión es si este lenguaje implica conceptualmente alguna noción de teleología o si se trata de un mero recurso retórico.

El fenómeno al que se asocian las explicaciones teleológicas es la adaptación. Dado que es el MESN el que explica dicho fenómeno, el debate puede enfocarse en si el modelo es o no teleológico, ya que si no lo fuera podría sostenerse que el darwinismo ha eliminado la teleología de la biología. El problema del presunto carácter teleológico del MESN será tratado en una sección posterior.

Los biólogos tienden a negar que la teleología ocupe un lugar legítimo en su ciencia y suelen afirmar que las expresiones teleológicas son solo un recurso retórico y metafórico. Así, por ejemplo, Futuyma (2009) sostiene que "... los conceptos de meta o propósito no tienen lugar en la biología [...]". Según Dawkins (2004) "... debemos tener presente que esta forma aparentemente teleológica de hablar [...] no es más que una especie de taquigrafía 
que puede traducirse fácilmente a términos darwinianos" y "Seguiré usando este lenguaje taquigráfico porque se adapta a nuestra forma natural de pensar". Esta postura que supone que las expresiones teleológicas podrían eliminarse es tal vez minoritaria en el ámbito de la filosofía de la biología, aunque es posible que predomine entre biólogos y profesores de esta materia. Algunos biólogos y filósofos (Mayr, 2006) reconocen que existen fenómenos biológicos dirigidos a fines pero prefieren hablar de "teleonomía" para evitar las presuntas connotaciones metafísicas indeseables de la teleología. Sin embargo, este concepto se basa en la noción de programa que es en sí misma teleológica, por lo que se trata de un cambio terminológico que no resuelve el problema de fondo (Ayala, 1970; Caponi, 2003; Mahner y Bunge, 2000; Ruse, 2003).

En síntesis, podemos concluir que, más allá del debate sobre su significado conceptual, la teleología ha persistido en el discurso de la biología tanto como su negación. Tal como señalan Mahner y Bunge (2000), “... los biólogos aparentemente no pueden vivir con la teleología, pero tampoco pueden vivir sin ella". Es imposible reseñar en el espacio disponible los extensos debates sobre este problema (véanse Allen, Bekoff y Lauder, 1998 y McLaughlin, 2003), por lo que nos limitaremos a explicitar nuestra postura en relación con el mismo. Partimos, como ya señalamos, de considerar que la persistencia del lenguaje teleológico en biología requiere una explicación. Señalar que dicho lenguaje es la expresión de una metáfora que se adopta en virtud de su poder heurístico o comunicativo no responde la pregunta planteada porque se puede replicar que, entonces, lo que demanda una explicación es por qué esta metáfora resulta tan útil (¿̇imprescindible?) para la biología. Siguiendo a Ruse (2003), sostendremos que la biología recurre a razonamientos y lenguaje teleológicos porque los organismos, a diferencia de las demás entidades naturales, parecen diseñados (Dawkins, 1998) y que dicho diseño aparente es consecuencia de que son producto de la selección natural. Por esta razón la MD es adecuada para el análisis de los organismos (al menos de sus rasgos adaptativos). Por otro lado, argumentaremos que el modelo científico que permite explicar este diseño biológico, el MESN, se basa necesariamente en la MD, por lo que es en sí mismo, en algún sentido relevante, teleológico. En la siguiente sección analizamos con más detenimiento esta cuestión.

\section{La naturaleza teleológica del modelo de evolución por selección natural}

Diferentes autores (Ayala, 1970; Brandon, 1981; Caponi, 2003; Dennett, 1995; Depew, 2008; Lennox, 1993; Ruse, 2008, 2003, 2000; Short, 2002 y Walsh, 2006) han convergido, a partir de diversos argumentos, en la conclusión de que las explicaciones darwinianas son, en algún sentido, teleológicas. Aquí reseñaremos solo dos análisis que nos parecen especialmente claros y útiles como fundamento para ofrecer una propuesta didáctica en relación con este problema. 
Caponi (2003) sostiene que las explicaciones basadas en el MESN son teleológicas debido a que se basan en el par categorial problema-solución. A diferencia de las explicaciones de otras subdisciplinas biológicas, como por ejemplo la fisiología, las explicaciones darwinianas no suponen identificar las causas físicas, eficientes, que, por así decirlo, "obligan" a que se produzca, como una consecuencia mecánica, el fenómeno que se intenta explicar (en nuestro caso, el cambio evolutivo adaptativo). Consisten, en cambio, en identificar "la razón de ser" de la variante seleccionada, en términos de por qué dicha variante es una mejor solución que las variantes alternativas en relación con cierto problema. El "problema" es cualquier factor (la temperatura, los depredadores, etc.) que limita las probabilidades de sobrevivir y reproducirse del organismo. Estas explicaciones son perfectamente compatibles con el supuesto de que todo lo que sucede es consecuencia de causas físicas eficientes, solo que la explicación darwiniana no consiste en identificar dichas causas. Otro aporte de Caponi (2003), especialmente interesante para la didáctica, consiste en la especificación de que el "nexo teleológico" en el MESN reside en la relación entre el factor selectivo y la mayor frecuencia de cierta variante en la población. Esta precisión es de interés porque en las concepciones de los estudiantes el nexo teleológico reside en la relación entre el problema y el origen de la variabilidad (González Galli y Meinardi, 2015).

El otro análisis que reseñaremos, y que nos servirá de base para nuestra propuesta didáctica, se debe a Michael Ruse $(2008,2003$, 2000). De acuerdo con este autor, aun en caso de que se considere que las explicaciones darwinianas no son formal y estructuralmente teleológicas, no es posible construir dichas explicaciones sin recurrir primero a la MD. Tal como hemos mencionado, según algunos autores, el MESN no es teleológico y el lenguaje teleológico es consecuencia del uso de la MD. El supuesto de que los términos teleológicos de la biología son metafóricos es discutido. Lewens (2004) menciona tres razones a favor de la interpretación metafórica: (1) Ha habido poco cambio en el uso que los biólogos hacen del lenguaje teleológico en los últimos dos siglos, (2) los términos teleológicos son más frecuentes en textos de divulgación y suelen ser usados entre comillas y (3) aunque los biólogos usan con frecuencia los términos teleológicos en su habla informal, no es frecuente encontrarlos en las publicaciones técnicas. Algunos autores consideran que esta metáfora se utilizaría con fines meramente heurísticos o comunicativos y que, por lo tanto, sería eliminable (Mahner y Bunge, 2000; Pramling, 2009). Ruse (2008, 2003, 2000), por el contrario, sostiene que el MESN es teleológico porque implica un recurso necesario a la MD. Así, el biólogo darwiniano observa el organismo o el rasgo cuya evolución quiere explicar como si de un objeto de diseño se tratara (Lewens, 2004, 2000) para, de ese modo, inferir a qué fin podría servir el rasgo de interés. Esta actitud analítica le permite formular hipótesis adaptacionistas que luego pondrá a prueba mediante diversas metodologías. En caso de que las evidencias favorezcan alguna hipótesis adaptacionista el investigador construirá luego un relato explicativo darwiniano. En dicho relato, el estado actual del rasgo se presenta como una consecuencia de ciertos acontecimientos del pasado sin que haya, al menos en apariencia, un razonamiento teleológico implicado. Sin embargo, sostiene Ruse, la construcción de este relato fue posible, en primer término, por el recurso a la MD que ahora, una vez alcanzado el objetivo, se elimina y se oculta. Así, dice Ruse (2000), usamos la metáfora del diseño con todas sus connotaciones teleológicas y luego pretendemos no haberla utilizado. De 
acuerdo con este autor, el recurso a esta metáfora es necesario, en el sentido de obligado. No hay modo de construir hipótesis darwinianas sobre la evolución de un rasgo sin plantear preguntas teleológicas derivadas de la MD: ¿̇Para qué sirve esa variante de un rasgo? ¿̇Qué función ${ }^{3}$ cumple? ¿ Qué ventaja confiere a su poseedor? ¿Qué problema soluciona? ¿Por qué es mejor que las demás variantes? (resaltamos en itálica los términos teleológicos en estas preguntas).

La evaluación que se haga del análisis de Ruse dependerá en parte de cómo se entienda el rol de las metáforas en relación con las teorías científicas. Considerando que esta cuestión tiene, además, gran relevancia para la enseñanza de las ciencias en general dedicamos la siguiente sección a reseñar las principales posturas al respecto.

\section{Las metáforas en la ciencia}

La terminología en relación con este tema no es unívoca. Por ejemplo, existen diversas propuestas para distinguir metáforas de analogías. Una tendencia general supone atribuir al término "metáfora" un alcance general para referirse a toda comparación y restringir el término "analogía" para aquellos casos en que se explicitan los aspectos y sentidos en que las dos entidades comparadas se asemejan (Aubusson, Harrison y Ritchie, 2006). En este artículo utilizaremos el término analogía siempre que nos refiramos a cuestiones didácticas porque nos plantearemos el objetivo de que los estudiantes lleven a cabo un análisis detallado y explícito de la MD. Cuando nos refiramos al ámbito de la biología evolutiva, utilizaremos indistintamente los términos analogía y metáfora. En cualquier caso, se trata de recurrir a una entidad conocida para facilitar la comprensión de otra más compleja o menos conocida. Ambas entidades se parecen en cierto sentido y grado y al mismo tiempo son diferentes en ciertos sentidos (de lo contrario se trataría de una identidad). Así, ciertos rasgos del objeto conocido son "mapeados sobre", "transferidos a" o "relacionados con" ciertos rasgos del objeto desconocido. El objeto familiar constituye el "campo base" o "campo fuente" y el desconocido el "campo blanco".

En relación con nuestros objetivos conviene distinguir tres planos en los que se pueden analizar las metáforas y analogías: psicológico (¿̇qué rol cumplen las analogías en el razonamiento?), epistemológico (żqué rol cumplen las analogías en la construcción y el funcionamiento de las teorías científicas?) y didáctico (¿̇qué rol cumplen las analogías en el aprendizaje?). En el ámbito de la psicología existe un amplio consenso sobre el hecho de que los razonamientos analógicos constituyen una estrategia cognitiva central, y no un mero recurso retórico, y que desempeñan un rol principal en los procesos de aprendizaje. En efecto, cuando

3 Aunque aquí asumimos que el concepto de función es teleológico debemos señalar que esta cuestión es objeto de debate. 
intentamos dar cuenta de una nueva situación o fenómeno tendemos a relacionarlo, mediante un procedimiento analógico, con situaciones o fenómenos ya conocidos que se le parezcan en cierto sentido (Aubusson et ál., 2006). Por otro lado, el lenguaje en particular está en gran medida basado en metáforas (Lakoff y Johnson, 1980; Pinker, 2007) ${ }^{4}$. En palabras del psicolingüista Steven Pinker (2007): ". . las personas no pueden colocar dos palabras juntas sin recurrir a las alusiones y las alegorías...".

En el plano epistemológico, en cambio, se encuentran diversas posturas en relación con el rol de las metáforas en la ciencia, aunque existe un amplio consenso en que los científicos recurren con frecuencia a las metáforas (Fox Keller, 2000; Thagard, 1995). Los disensos pueden plantearse identificando dos posturas extremas. Así, algunos autores consideran que las metáforas son un mero recurso retórico o heurístico (y por lo tanto son eliminables) mientras que para otros son una parte constitutiva de las teorías (y, por lo tanto, no eliminables). Desde el positivismo lógico se ha considerado que explicar consiste en utilizar generalizaciones lógicas (leyes) y relaciones deductivas literales entre el lenguaje teórico y el observacional. Así, como señala Hoffman (1980), la metáfora constituye en el mejor de los casos una mera herramienta heurística y en el peor "un irreal hongo nefasto condenado eternamente a una dimensión desconocida precientífica". En la misma línea, Fodor (1996) ha escrito que "Cuando realmente comienzas a hacer ciencia, las metáforas se abandonan y las estadísticas toman su lugar". Desde esta

Aunque el lenguaje cumple una función importantísima en el pensamiento, la hipótesis de que "pensamos con el lenguaje" no goza de mucho apoyo en la actualidad (Dennet, 1995; Pinker, 2001) y es objeto de discusión qué relación hay entre el hecho de que el lenguaje sea principalmente metafórico con el problema de cómo pensamos (Pinker, 2007). perspectiva las metáforas no tienen (o no deberían tener) lugar en la ciencia, que debería basarse exclusivamente en el lenguaje literal (Bradie, 1999; Ortony, 1993). Como señala Ciapuscio (2003), desde este enfoque se ha censurado a la metáfora "por considerarla un recurso distorsionador, intrínsecamente ambiguo, propio del campo subjetivo y emocional" y, continúa esta autora, "Subyace a esta valorización la concepción clásica de la metáfora como 'ornamento lingüístico' y, en última instancia, como mera sustitución de palabras". Esta postura rígida, sin embargo, ha ido perdiendo apoyo a la luz de numerosos análisis que parten de reconocer que la ciencia es una actividad social y que, como tal, está fuertemente influida por la cultura general, incluido el lenguaje. En este sentido, desde el constructivismo epistemológico se asigna un rol más importante a las metáforas en la ciencia y, de hecho, el reconocimiento de que no tenemos un conocimiento directo de la realidad física tiende a desdibujar la distinción entre lenguaje literal y metafórico (Ortony, 1993). Otro factor que tendió a minar la postura positivista fue el reconocimiento, ya mencionado, de que las analogías no son solo un mero recurso retórico sino que son también un instrumento del pensamiento. Así, hay un consenso creciente en afirmar que la ciencia se hace con metáforas (Ciapuscio, 2003).

Este problema se relaciona, también, con las distintas perspectivas sobre la naturaleza de las teorías científicas. Desde la perspectiva positivista las metáforas no son parte de las teorías y, por lo tanto, son eliminables sin que dicha eliminación suponga una pérdida de significado. El enfoque positivista asume la llamada perspectiva sintáctica de las teorías científicas de acuerdo con la cual las teorías son conjuntos de afirmaciones (algunas de las cuales expresan leyes) sobre cierto ámbito de la realidad o "cálculos interpretados" (Díez y 
Moulines, 2008). Por el contrario, desde las perspectivas semánticas se propone que lo que define a una teoría son los modelos que la conforman (y no algún conjunto de enunciados $u$ otra entidad lingüística). Estos modelos dicen algo sobre algún aspecto del mundo y lo que importa es el significado, es decir qué dicen sobre el mundo. Desde esta perspectiva, la metáfora supone una sustitución (o una interacción) de significados (Palma, 2007), por lo que difícilmente puede "decirse lo mismo" tras eliminar la metáfora (Bradie, 1999). Así, lo que una teoría dice sobre el mundo (y esto es lo importante para la perspectiva semántica) dependerá -al menos parcialmente- de las metáforas a las cuales recurra. Si consideramos además que muchos autores sostienen que la aplicación de las teorías científicas, es decir, la acción de dar cuenta de ciertos fenómenos desde la teoría, supone inevitablemente el uso de metáforas (Bradie, 1999) debemos concluir que las metáforas asumen un rol central y constitutivo en la ciencia.

Adoptamos entonces en este trabajo la perspectiva según la cual las metáforas constituyen un aspecto central e intrínseco de la cognición humana y, más específicamente, de las teorías científicas. Así, si bien cabe distinguir diferentes funciones de las metáforas particulares en relación con las teorías científicas 5 , no es concebible la investigación científica como un proceso que, de acuerdo con ciertos ideales positivistas, pueda basarse enteramente en un lenguaje literal, prescindiendo de todo recurso a la metáfora. Creemos que esta conclusión tiene implicancias directas para el problema que nos ocupa.

\section{Las metáforas en biología evolutiva}

La biología evolutiva es rica en metáforas (Shelley, 1999; Villamar et ál., 2007). Darwin, en particular, hizo un uso extensivo de las metáforas (Gould, 2004, Noguera Solano, 2013; Ruse, 2008, 2003). Algunas de las metáforas de la biología evolutiva han recibido mucha atención tanto por parte de filósofos y biólogos como de educadores y didactas (Pramling, 2009). Por ejemplo, la metáfora según la cual el proceso de diversificación filogenético es como un árbol (Archibald, 2014; Pietsch, 2012) y la analogía entre la selección artificial y la selección natural (Evans, 1984; Gildenhuys, 2004; Sterrett, 2002; Theunissen, 2012; Wilner, 2006) han sido muy analizadas. Aunque quizás en menor medida, la MD también ha recibido bastante atención por parte de los filósofos de la biología (véase, por ejemplo, Dennett, 1995; Lewens, 2004, 2000; Noguera Solano, 2013; Ruse, 2003). Sin embargo, en el ámbito de la didáctica, la MD ha sido sistemáticamente ignorada. Sugerimos dos posibles razones (que no son totalmente independientes) para esta evitación. Por un lado, la idea de diseño se asocia (correctamente) con la teleología y dada la "perspectiva tradicional" frente a este problema es de esperar que se considere que la MD es ilegítima y que, por lo

5 Se ha sugerido que las metáforas cumplen al menos tres funciones (Bradie, 1999; Ruse, 1994): heurística, retórica y cognitiva. Thagard (1995), por su parte, distingue cuatro funciones: descubrimiento, desarrollo, evaluación y exposición. 
tanto, lo mejor es evitarla. Por otro lado, cabe especular que el reconocimiento explícito del rol de esta metáfora podría verse dificultado por el problemático debate entre evolucionismo y creacionismo. Tal vez, los evolucionistas, en especial aquellos comprometidos con la educación, temen que el reconocimiento de que el darwinismo supone alguna continuidad con la teología natural de Paley en virtud del mantenimiento de esta metáfora (Ruse, 2003) pueda ser utilizado retóricamente por el autodenominado "creacionismo científico" (o por su más reciente versión, la "teoría del diseño inteligente") para cuestionar el rigor científico de la TE. Tal podría ser el caso de Dawkins (1998), quien distingue los objetos que son producto del azar de aquellos que son producto del diseño y sostiene que los organismos constituyen una tercera categoría de objetos que denomina "diseñoides". Estos objetos, dice Dawkins, "han sido construidos por un magnífico proceso no aleatorio que crea una ilusión casi perfecta de diseño". Es claro, a partir de este párrafo, que este autor piensa los organismos como si fueran objeto de diseño o, lo que es lo mismo, que está recurriendo a la MD. Sin embargo, ofrece el neologismo "diseñoide" para evitar el término "diseño" (Dawkins, 1998).

\section{Las metáforas en la enseñanza de las ciencias}

Según lo dicho en la sección anterior consideramos que no sería defendible evitar en el ámbito de la enseñanza de las ciencias un recurso cognitivo y expresivo que parece ocupar un rol central en la propia ciencia. Por otro lado, desde el enfoque de la naturaleza de la ciencia (Erduran y Dagher, 2014), cabe señalar que además de enseñar ciencia siempre enseñamos sobre ciencia, es decir, implícita e inconscientemente o -lo que sería deseable- explícita y conscientemente contribuimos a que los estudiantes construyan una determinada imagen de ciencia y de científico. En este sentido, aprender acerca del rol de las metáforas ayudaría a comprender qué es la ciencia y cómo se construye. Por otro lado, es un hecho que los profesores de ciencia-aun los que no las consideran deseables- recurren sistemáticamente a las analogías (Linares e Izquierdo Aymerich, 2006). En síntesis, consideramos que no es posible evitar las analogías en la enseñanza de las ciencias. Así, la cuestión pasa a ser cómo usarlas (Aubusson et al., 2006; Brown y Clement, 1989; Clement, 1993; Dagher, 1995; Linares e Izquierdo Aymerich, 2006; Oliva, 2004; Oliva Martínez y Aragón Méndez, 2009, Oliva, Aragón, Mateo y Bonat, 2001, entre otros). Los modos en que los profesores recurren a las analogías son variados (Dagher, 1995) y, tal como señala Oliva (2004), lo más frecuente es que aparezcan como parte de una explicación a cargo del profesor, sin involucrar a los estudiantes en actividades que supongan un trabajo cognitivo más complejo. También señala este autor que con frecuencia la analogía se presenta más como otro contenido estático por aprender que como un proceso que implique la construcción o reconstrucción de la analogía. Dos conclusiones centrales de muchas propuestas sobre el uso de analogías en la enseñanza son la necesidad del tratamiento explícito de las mismas y de una reflexión metacognitiva sobre su uso y de un trabajo cognitivo por parte de los estudiantes basado en la analogía en cuestión. 


\section{La metáfora del diseño en biología y la naturaleza teleológica del MESN: implicancias y propuestas didácticas}

Lo primero que debemos decir es que si asumimos la postura epistemológica de acuerdo con la cual el MESN es teleológico y aquella que supone un rol central para las metáforas en la ciencia no podemos sostener la "perspectiva tradicional" en relación con el problema de la teleología. Esto no implica, sin embargo, negar que las intuiciones teleológicas de los estudiantes puedan, en ciertos momentos, dificultar el aprendizaje: ya mencionamos, al reseñar el análisis de Caponi, que las concepciones de los estudiantes y el MESN son teleológicas pero no en el mismo sentido. Es más, tal como hemos sostenido en otros trabajos (González Galli y Meinardi, 2015 y 2011), y en consonancia con muchas otras investigaciones (Jiménez Aleixandre, 1991 y Settlage, 1994), consideramos que este tipo de razonamiento constituye un obstáculo para el aprendizaje del MESN. Es, de hecho, uno de los principales factores que hacen que la teoría de la evolución sea fuertemente contraintuitiva y, por lo tanto, difícil de comprender (Kelemen, 2012). Reconocemos, por lo tanto, la necesidad de tener en cuenta este problema a la hora de enseñar al tiempo que diferimos en el modo tradicional de tratarlo y proponemos un modo alternativo de hacerlo basado en un trabajo explícito sobre la MD. Aunque hemos defendido la perspectiva según la cual la MD es constitutiva de las explicaciones darwinianas, creemos que las implicancias didácticas que derivamos de estos análisis son las mismas en caso de que, sobre bases más moderadas, se considere que aunque eliminable en teoría la MD no lo es en la práctica, sea por cuestiones "estratégicas" (su valor heurístico o comunicativo) (Zohar y Ginossar, 1998) o porque se trata de un estilo de pensamiento muy arraigado en la estructura cognitiva de los estudiantes (Kelemen, 2012).

Dadas las sugerencias sobre el uso de analogías que hemos reseñado y aquellas derivadas del marco teórico de los obstáculos epistemológicos (Peterfalvi, 2001) la noción de metacognición emerge como central. En efecto, numerosas investigaciones convergen en que se trata de uno de los principales factores que condicionan los procesos de aprendizaje (Campanario, 2000; Hacker Dunlosky y Graesser,2009; Peña Ayala, 2015; Zohar y Yehudit Dori, 2012). Uno de los riesgos del uso de analogías en educación reside en que los estudiantes podrían tomar la analogía como una identidad. Jungwirth (1975a), quien consideró que la teleología era consecuencia de la metáfora antropomórfica, señaló este riesgo en el caso que nos ocupa. Consideramos que el modo de evitar este problema es llevar a cabo un tratamiento explícito de la MD. Basándonos en varios análisis, proponemos que la metacognición puede entenderse como el conocimiento sobre los procesos cognitivos en general, sobre los propios procesos cognitivos y la capacidad de control y regulación de dichos procesos. Así, nuestros objetivos son que los estudiantes:

1. Comprendan en qué consiste una metáfora en general y la MD en particular (conocimiento general sobre cognición) y puedan reconocerla. 
2. Tomen conciencia sobre el rol de la MD en sus propios procesos cognitivos (conocimiento sobre la propia cognición), es decir, que puedan reconocer que ellos recurren (normalmente de un modo implícito e inconsciente) a la MD.

3. Puedan ejercer un control consciente sobre el recurso a la MD, esto es, que puedan decidir, de un modo adecuado al contexto, cuándo y cómo utilizarla (control y autorregulación).

Esta propuesta supone la capacidad de distinguir las expresiones metafóricas de las literales. La cuestión de en qué medida los estudiantes pueden alcanzar esta capacidad en relación con esta metáfora en particular ha sido poco estudiada (Jungwirth, 1975a; Tamir y Zohar, 1991) y las evidencias disponibles sugieren que dicho objetivo es complejo pero alcanzable. A continuación proponemos, a modo de ejemplo, algunas actividades tendientes a alcanzar los objetivos enunciados. Lo que presentamos no es una unidad didáctica sino solo tres actividades con el objeto de ilustrar posibles modos de trabajar la MD. Este tipo de actividades deberían llevarse a cabo después de haber trabajo qué es la teleología. Algunas actividades para el trabajo sobre el pensamiento finalista pueden verse en González Galli, Revel Chion y Meinardi (2008). Aunque ninguna actividad por sí sola puede dar cuenta totalmente de ninguno de estos objetivos, ya que se trata de logros a mediano plazo, cada actividad está enfocada en alguno de los objetivos específicos enunciados.
Actividad 1. "La metáfora del diseño en los escritos de Darwin". Comprender en qué consiste la metáfora del diseño y cómo reconocerla

Los textos de Darwin constituyen un recurso de gran utilidad para comprender qué es una metáfora en general y, más específicamente, en qué consiste la MD. Algunos ejemplos de expresiones de la MD en los textos de Darwin ${ }^{6}$ son:

- "El significado o uso de la existencia en Primula de las dos formas en número aproximadamente iguales [...] es medianamente claro; a saber, favorecer el cruzamiento entre individuos distintos. Con las plantas existen innumerables artilugios (contrivances) para este fin; y nadie podrá comprender que la causa final de la estructura de muchas flores sin atender a este fin" (Darwin, 1862a).

- "Aunque un órgano podría no haber sido originalmente formado para un propósito especial, si ahora sirve para este fin, estamos justificados para decir que está especialmente adaptado para él" (Darwin, 1862b).

- "La naturaleza -si se me permite personificar la conservación o supervivencia natural de los más adecuados- no atiende a nada por las apariencias, excepto en la medida que son útiles a los seres" (Darwin, 2009).

- "Metafóricamente, puede decirse que la selección natural está buscando cada día y cada hora por

Las versiones en castellano de todas las citas (excepto las de Darwin 2009) son del autor. En la sección "Referencias bibliográficas" el lector encontrará las citas para acceder a los textos originales, completos y en inglés. 
todo el mundo las más ligeras variaciones; rechazando las que son malas; conservando y sumando todas las que son buenas; trabajando silenciosa e insensiblemente [...] por el perfeccionamiento de cada ser orgánico en relación con sus condiciones orgánicas e inorgánicas de vida" (Darwin, 2009).

- “... en condiciones naturales, la selección natural regula hasta la más insignificante diferencia. Así como las piedras cuadradas, o los ladrillos - las vigas son los materiales indispensables para una construcción e influyen su naturaleza; así la variabilidad no solo es indispensable, sino también influyente; más aún, del mismo modo, así como el arquitecto es una persona importantísima en una construcción, así lo es la selección con los cuerpos orgánicos" (Darwin, 2015a).

- "He dicho que la selección natural es a la estructura de los seres organizados lo que el arquitecto humano es a la construcción" (Darwin, 2015b).

- "Una vez que varias razas se han formado, su entrecruzamiento ayuda al progreso de la modificación, y puede incluso producir nuevas razas. Pero, como en la construcción de un edificio, las meras piedras o ladrillos son de poca utilidad sin el arte del constructor, así, en la producción de nuevas razas, la selección ha sido el poder principal" (Darwin, 1968).

- "Fuerza a un arquitecto a construir un edificio con rocas enteras, caídas en un precipicio. La forma de cada fragmento podría calificarse de accidental; aún así la forma de cada uno ha sido determinada por la fuerza de la gravedad, la naturaleza de la roca, y la pendiente del precipicio -eventos y circunstancias, todos los cuales dependen de leyes naturales-; pero no hay relación entre estas leyes y el propósito por el cual cada fragmento es usado por el constructor" (Darwin, 1968).

También se podrían utilizar ejemplos de cualquier texto de biología, ya que aun en libros universitarios actuales son frecuentes las expresiones teleológicas que se pueden interpretar fácilmente como expresiones de la MD. El uso de los textos de Darwin, sin embargo, tiene un valor agregado dado por el hecho de que permite poner en evidencia que el uso de las metáforas es central en la elaboración de las teorías científicas.

Algunas preguntas que podrían orientar la discusión a partir de estos textos son:

1. ¿̇Qué tienen en común todos estos textos?

2. ¿̇Cómo describirían el tipo de razonamiento que subyace a todos los textos?

3. ¿En qué consiste la analogía que Darwin está estableciendo? Identifiquen el "campo fuente" y el "campo blanco". 
4. ¿̇Con qué objetivo/s creen que Darwin introdujo esta analogía en sus textos?

Las preguntas (1) y (2) permitirían al docente introducir el concepto de analogía con algunos elementos de análisis específicos como la distinción entre "campo fuente" y "campo blanco". La pregunta (3) apunta a identificar la MD y la (4) a discutir los posibles usos (retórico, heurístico, justificativo) de la misma que hizo Darwin. La discusión de esta actividad permitiría llegar a un consenso de acuerdo con el cual lo que resulta semejante entre las adaptaciones y los artefactos (o entre la selección natural y el diseño deliberado) es que en ambos casos el resultado son estructuras y procesos especialmente adecuados a un fin o función. La principal diferencia sería que en un caso (selección natural) esto es resultado de un proceso no dirigido intencionalmente ni previsor mientras que en el otro (diseño deliberado) sería el resultado de un proceso intencionalmente dirigido.

Actividad 2. "La metáfora del diseño en los escritos propios". Identificar la metáfora del diseño en las propias producciones para tomar conciencia de cómo se piensa

A fin de ayudar a los estudiantes a identificar expresiones de las MD en sus propias producciones se les pueden plantear algunas preguntas que demanden explicar un caso evidente de evolución adaptativa, como podría ser el mimetismo o la evolución de las membranas interdigitales en las patas de las aves nadadoras. Lo más probable es que produzcan textos basados en algunas concepciones que han sido ampliamente analizadas en la investigación en didáctica y que suelen suponer que son los individuos los que se transforman adaptativamente y que luego estas transformaciones son heredadas por la descendencia (González Galli y Meinardi, 2015; Jiménez Aleixandre, 1991). En estas explicaciones abundan las expresiones teleológicas, muchas de las cuales podrán interpretarse fácilmente como expresiones de la MD.

Algunas preguntas que podrían guiar la discusión a partir de los textos resultantes de esta actividad son:

1. ¿PPueden tus respuestas considerarse teleológicas? ¿Cuáles podrían interpretarse como expresiones de la MD?

2. ¿Cómo las reconociste?

3. ¿̇as consideras adecuadas o cambiarías algo en el texto?

La idea es que el problema de evolución (el caso de mimetismo o equivalente) se resuelva antes de la actividad 1 (o de alguna equivalente). Luego, durante la actividad 1, los estudiantes aprenderán en qué consiste la MD (ya habían aprendido qué es la teleología) y recién después podrán analizar sus producciones en busca de expresiones teleológicas que puedan considerase expresiones de la MD.

Actividad 3. "¿¿Cuándo y cómo usar la metáfora del diseño?" Comprender en qué casos y en qué sentido el recurso a la metáfora del diseño es adecuado

En este caso una estrategia posible es dar a los estudiantes diversos textos en los que se utiliza la MD y pedirles que los evalúen críticamente. No se trata ahora solo de reconocer la MD sino, además, de evaluar su adecuación. Por ejemplo, se pueden comparar estos dos enunciados: 
1. "La selección natural es como un artesano que busca construir una obra y modifica y une los materiales para alcanzar ese fin".

2. "La selección natural es como un artesano que a partir de ciertos materiales construye obras más complejas y adecuadas a fines específicos".

Podría considerarse que en el primer caso la MD está mal aplicada porque se afirma una similitud entre ambos campos que no es legítima: la capacidad de previsión y la intencionalidad. Por el contrario, en el segundo solo se afirma la similitud que da sentido a la analogía: el carácter funcional del producto. Podría discutirse, a partir de este caso, la conveniencia de explicitar aquellos aspectos en los que ambos campos semánticos no se parecen. La expresión "el relojero ciego" fue acuñada por Richard Dawkins (1988) para referirse a la selección natural y busca poner en evidencia la diferencia señalada entre los casos (1) y (2): al igual que un artífice, la selección genera objetos de aparente diseño, pero lo hace "a ciegas", sin intención, conciencia ni premeditación. La discusión de esta expresión podría ser parte de esta actividad dedicada a comprender los alcances de la analogía y sus limitaciones.

Otro criterio que se debe tener en cuenta para el análisis de la adecuación de la MD es que solo sería pertinente en casos en que, al menos hipotéticamente, se considere que el rasgo analizado es adaptativo. Cabe recordar que la evolución puede proceder por mecanismos no selectivos (por ejemplo, la deriva génica) y que tales casos no los explica el MESN (Futuyma, 2009), motivo por el cual la MD no sería pertinente. Es decir, la teleología está asociada a la adaptación (y a conceptos relacionados como el de función) y al modelo que la explica (el MESN) y no a la biología en general.

\section{Conclusiones}

El sesgo teleológico del razonamiento de los estudiantes en relación con los seres vivos es un factor que dificulta la comprensión del MESN. La "perspectiva tradicional" frente a este problema ha supuesto censurar las expresiones de dichos razonamientos. Desde una perspectiva diferente hemos caracterizado, en otros trabajos, el pensamiento teleológico como un obstáculo epistemológico (González Galli y Meinardi, 2015) y, de acuerdo con este marco teórico, hemos sugerido un tratamiento didáctico centrado en el desarrollo de una "vigilancia" metacognitiva de los estudiantes sobre este tipo de razonamiento (Peterfalvi, 2001). Por otro lado, en la propia biología se encuentra una situación ambigua respecto de la teleología y el reconocimiento de este rasgo epistemológico demanda una reconsideración de las estrategias didácticas. Siguiendo a Michael Ruse, en este trabajo hemos sugerido que puede ser útil considerar que el recurso al lenguaje y el razonamiento teleológico en la biología es una consecuencia de que los biólogos recurren a la MD cuando analizan los rasgos adaptativos de los organismos. En palabras de Ruse (2003): "La metáfora del diseño, con el 
organismo como artefacto, está en el corazón de la biología evolutiva darwiniana" y

... Diseño. Es por lo que el lenguaje teleológico se considera apropiado. Las adaptaciones son como artefactos. Hablamos de fines cuando nos referimos a objetos hechos por humanos, así analógicamente [o metafóricamente] hablamos de fines cuando nos referimos a adaptaciones. Si el corazón no fuera como una bomba [hecha por humanos], el lenguaje teleológico nunca surgiría, pero lo es y por eso surge el lenguaje teleológico.

Nos hemos basado en esta consideración epistemológica para sugerir que el tratamiento didáctico de este problema podría centrarse en el análisis explícito de la MD y hemos sugerido algunas actividades dirigidas a este objetivo. El valor de estas sugerencias solo podrá evaluarse en la medida en que se lleven a la práctica intervenciones concretas en las aulas basadas en los criterios sugeridos. Creemos que este puede ser un modo innovador para abordar el antiguo problema del finalismo en la enseñanza de la biología.

Para terminar, queremos retomar el texto pionero de Jungwirth (1975b) en el que, en referencia a quienes sostienen la "perspectiva tradicional" sobre este problema, señala que

Cuesta creer que los autores mencionados no estén al tanto del carácter controversial de sus afirmaciones, o que deliberadamente elijan ignorarlo. Si no están al tanto, sería más deseable que, como otros, omitan enteramente hacer referencia a la teleología y sus ramificaciones. Si su actitud es deliberada, las implicancias son más serias, porque en tal caso estaría justificado el término "indoctrinación".

Cuarenta años después de esta atinada observación crítica de Jungwirth el panorama no parece haber cambiado mucho. Esperamos que este artículo contribuya en alguna medida a revisar la "perspectiva tradicional" sobre el problema de la teleología en la enseñanza.

\section{Referencias bibliográficas}

Allen, C. (2003). Teleological notions in biology. En E. Zalta, (ed.). The Stanford Encyclopedia of Philosophy (Winter, 2003 ed.). Recuperado de http://plato. stanford.edu/entries/teleology-biology/.

Allen, C.; Bekoff, M. y Lauder, G. (1998). Nature's purposes. Analyses of function and design in biology. Cambridge, EUA: MIT Press.

Archibald, J. (2014). Aristotle's ladder, Darwin's tree. The evolution of visual metaphors for biological order. Nueva York: Columbia University Press.

Astolfi, J. (1999). El "error", un medio para enseñar. Sevilla: Díada.

Astolfi, J. y Develay, M. (1989). La didactique des sciences. París: Presses Universitaires de France.

Aubusson, P.; Harrison, A. y Ritchie, S. (2006). Metaphor and analogy in science education. Dordretch, Holanda: Springer. 
Ayala, F. (1970). Teleological explanations in evolutionary biology. Philosophy of Sciences, 37(1), 1-15.

Bachelard, G. (2004). La formación del espíritu científico. Contribución a un psicoanálisis del conocimiento objetivo. México: Siglo xxl.

Bishop, B. y Anderson, C. (1990). Students' conceptions of natural selection and its role in evolution. Journal of Research in Science Teaching, 27(5), 415-427.

Bradie, M. (1999). Science and metaphor. Biology and Philosophy, 14(2), 159-166.

Brandon, R. (1981). Biological teleology: Questions and explications. Studies in History and Philosophy of Science, 12(2), 91-105.

Brown, D. y Clement, J. (1989). Overcoming misconceptions via analogical reasoning: abstract transfer versus explanatory model construction. Instructional Science, 18(4), 237-261.

Campanario, J. (2000). El desarrollo de la metacognición en el aprendizaje de las ciencias: estrategias para el profesor y actividades orientadas al alumno. Enseñanza de las Ciencias: Revista de Investigación y Experiencias Didácticas, 18(3), 369-380.

Caponi, G. (2003). Darwin: entre Paley y Demócrito. História, Ciências, Saúde. Manguinhos, 10(3), 993-1023.

Chediak, K. (2006). Análise do conceito de função a partir da interpretação histórica. Filosofia e História da Biologia, 1, 161-174.

Chediak, K. (201 1). Função e explicações funcionais em Biologia. En P. Abrantes (org.). Filosofia da Biologia. Porto Alegre, Brasil: Artmed.

Ciapuscio, G. (2003). Metáforas y ciencia. Ciencia Hoy, 76(13), 60-66.

Clement, J. (1993). Using bridging analogies and anchoring intuitions to deal with student's preconception in physics. Journal of Research in Science Teaching, 30(10), 1241-1257.

Dagher, Z. (1995). Analysis of analogies used by science teachers. Journal of Research in Science Teaching, 32(3), 259-270.

Darwin, C. (1862a). On the two forms, or dimorphic condition, in the species of Primula, and on their remarkable sexual relations. Journal of the Proceedings of the Linnean Society of London (Botany), 6, 77-96. Recuperado de http:// darwin-online.org.uk/converted/published/1862_primula_F1717.html

Darwin, C. (1862b). On the various contrivances by which British and foreign orchids are fertilised by insects and on the good effects of intercrossing. Londres: John Murray. Recuperado de http://darwin-online.org.uk/content/frameset? pagese$q=1$ \&itemID $=F 800$ \&viewtype $=$ text 
Darwin, C. (1868). The variation of animals and plants under domestication (Vol. 2). Londres: John Murray. Recuperado de: http://darwin-online.org.uk/converted/ pdf/1868_Variation_F878.2.pdf

Darwin, C. (2009). El origen de las especies por medio de la selección natural. Madrid, España: Alianza.

Darwin, C. (2015a). Correspondence Database. Recuperado de http://www. darwinproject.ac.uk/entry-2832.

Darwin, C. (2015b). Correspondence Database. Recuperado de http://www. darwinproject.ac.uk/entry-2833.

Dawkins, R. (1988). El relojero ciego. Barcelona: Labor.

Dawkins, R. (1998). Escalando el monte improbable. Barcelona: Tusquets.

Dawkins, R. (2004). El cuento del antepasado. Un viaje a los albores de la evolución. Barcelona: Antoni Bosch.

Dennett, D. (1995). Darwin's dangerous idea: Evolution and the meanings of life. Nueva York: Simon and Schuster.

Depew, D. (2008). Consequence etiology and biological teleology in Aristotle and Darwin. Studies in History and Philosophy of Biology and Biomedical Sciences, 39(4), 379-390.

Díez, J. y Moulines, C. (1999), Fundamentos de filosofía de la ciencia. Barcelona: Ariel.

Dupré, J. (2006). El legado de Darwin. Qué significa hoy la evolución. Buenos Aires: Katz.

Erduran, S. y Dagher, Z. (2014), Reconceptualizing the nature of science. Scientific knowledge, practices and oher family categories. Dordretch, Holanda: Springer.

Estany, A. (1993), Introducción a la filosofía de la ciencia. Barcelona: Grijalbo-Mondadori.

Evans, L. (1984). Darwin's use of the analogy between artificial and natural selection. Journal of the History of Biology, 17(1), 113-140.

Fodor, J. (1996). Peacocking. London review of books. Recuperado de http://www. Irb.co.uk/v18/n08/jerry-fodor/peacocking.

Fox Keller, E. (2000), Lenguaje y vida. Metáforas de la biología en el siglo xx. Buenos Aires: Manantial.

Futuyma, D. (2009). Evolution. Sunderland, EUA: Sinauer.

Gaeta, R.; Gentile, N.; Lucero, S. y Robles, N. (1996). Modelos de explicación científica. Problemas epistemológicos de las ciencias naturales y sociales. Buenos Aires: Eudeba. 
Gildenhuys, P. (2004). Darwin, Herschel, and the role of analogy in Darwin's origin. Studies in History and Philosophy of Biology and Biomedical Sciences, 35(4), 593-611.

González Galli, L. y Meinardi, E. (2010). Revisión del concepto de obstáculo a partir de la investigación sobre la enseñanza y el aprendizaje del modelo de evolución por selección natural. Ix Jornadas Nacionales-iv Congreso Internacional de Enseñanza de la Biología. DOI: http://congresosadbia.com/ocs/index.php/ tucuman2010/tucu2010/paper/viewFile/496/317

González Galli, L. y Meinardi, E. (2011). The role of teleological thinking in learning the Darwinian model of evolution. Evolution: Education and Outreach, 4(1), 145-152.

González Galli, L. y Meinardi, E. (2015). Una investigación sobre los obstáculos para el aprendizaje del modelo de evolución por selección natural en estudiantes de escuela secundaria de Argentina. Ciencia y Educação, 21 (1), 101-122.

González Galli, L.; Revel Chion, A. y Meinardi, E. (2008). Actividades centradas en obstáculos para enseñar el modelo de evolución por selección natural. Revista de Educación en Biología, 17 (1), 52-55.

Gould, S. (2004). La estructura de la teoría evolutiva. Barcelona: Tusquets.

Hacker, D.; Dunlosky, J. y Graesser, A. (eds.). (2009). Handbook of Metacognition in Education. Nueva York: Routledge.

Hallden, O. (1988). The evolution of species: Pupil perspectives and school perspectives. International Journal of Science Education, 10(5), 541-552.

Jiménez Aleixandre, M. (1991). Cambiando las ideas sobre el cambio biológico. Enseñanza de las Ciencias: Revista de Investigación y Experiencias Didácticas, 9(3), 248-256.

Jungwirth, E. (1975a). Caveat mentor-Let the teacher beware! Research in Science Education, 5(1), 153-160.

Jungwirth, E. (1975b). The problem of teleology in Biology as a problem of Biology-teacher education. Journal of Biological Education, 9(6), 243-246.

Kelemen, D. (2012). Teleological minds: How natural intuitions about agency and purpose influence learning about evolution. En K. Rosengren; S. Brem, E. Evans y G. Sinatra (eds.) Evolution challenges. Integrating research and practice in teaching and learning about evolution. Oxford, Inglaterra: Oxford University Press.

Lakoff, G. y Johnson, M. (2012). Metáforas en la vida cotidiana. Madrid: Cátedra. Lennox, J. (1993). Darwin was a teleologist. Biology and Philosophy, 8(4), 409-421.

Lewens, T. (2000). Function talk and the artefact model. Studies in History and Philosophy of Biology and Biomedical Sciences, 37 (1), 95-111. 
Lewens, T. (2004). Organisms and artifacts. Design in nature elsewhere. Cambridge, EUA: MIT Press.

Linares, R. e Izquierdo Aymerich, M. (2006). El rescate de la princesa encerrada en lo más alto de la más alta torre. Un episodio para aprender sobre analogías, símiles y metáforas. El Hombre y la Máquina, 27, 24-37.

Mahner, M. y Bunge, M. (2000). Fundamentos de biofilosofía. México D. F.: Siglo XXI.

Mayr, E. (2006). Por qué es única la biología. Consideraciones sobre la autonomía de una disciplina científica. Buenos Aires: Katz.

McLaughlin, P. (2003). What functions explain. Functional explanation and self-reproducing system. Cambridge, Inglaterra: Cambridge University Press.

Nagel, E. (2006). La estructura de la ciencia. Problemas de la lógica de la investigación científica. Barcelona: Paidós.

Noguera Solano, R. (2013). The metaphor of the architect in Darwin: Chance and free will. Zygon, 48(4), 859-874.

Nunes-Neto, N.; Carmo, R. y El-Hani, C. (2013). O conceito de função na Biologia contemporânea. Revista de Filosofia. Aurora, 25(36), 43-73.

Nunes-Neto, N. y El-Hani, C. (2009). O que é função? Debates na filosofia da Biologia contemporânea. Scientiae Studia, 7(3), 353-401.

Oliva, J. M.; Aragón, M. M.; Mateo, J. y Bonat, M. (2001). Una propuesta didáctica basada en la investigación para el uso de las analogías en la enseñanza de las ciencias. Enseñanza de las Ciencias: Revista de Investigación y Experiencias Didácticas, 19(3), 453-470.

Oliva, L. (2004). El pensamiento analógico desde la investigación educativa y desde la perspectiva del profesor de ciencias. Revista Electrónica de Enseñanza de las Ciencias, 3(3), 363-384.

Oliva-Martínez, J. y Aragón-Méndez, J. (2009). Contribución del aprendizaje con analogías al pensamiento modelizador de los alumnos en ciencias: marco teórico. Enseñanza de las Ciencias: Revista de Investigación y Experiencias Didácticas, 27(2), 195-208.

Ortony, A. (ed.). (1993). Metaphor and thought. Cambridge, Inglaterra: Cambridge University Press.

Palma, H. (2007). Metáforas en la evolución de las ciencias. Buenos Aires: Jorge Braulio Ediciones.

Peña Ayala, A. (ed.). (2015). Metacognition: fundaments, applications and trends. A profile of current state-of-the-art. Dordretch, Holanda: Springer. 
Peterfalvi, B. (2001), Identificación de los obstáculos por parte de los alumnos. En A. Camilloni (ed.). Los obstáculos epistemológicos en la enseñanza. Barcelona: Gedisa.

Pietsch, T. (2012). Trees of life. A visual history of evolution. Baltimore: Johns Hopkins University Press.

Pinker, S. (2001). Cómo funciona la mente. Barcelona: Destino.

Pinker, S. (2007). El mundo de las palabras. Una introducción a la naturaleza humana. Barcelona: Paidós.

Pramling, N. (2009). The role of metaphor in Darwin and the implications for teaching evolution. Science Education, 93(3), 535-547.

Rosenberg, A. y McShea, D. (2008). Philosophy of biology. A contemporary introduction. Nueva York: Routledge.

Ruse, M. (2000). Teleology: Yesterday, today, and tomorrow? Studies in History and Philosophy of Biological \& Biomedical Sciences, 37 (1), 213-232.

Ruse, M. (2003). Darwin and design. Does evolution have a purpose? Cambridge, EUA: Harvard University Press.

Ruse, M. (2008). Charles Darwin. Buenos Aires, Argentina: Katz.

Settlage, J. (1994). Conceptions of natural selection: A snapshot of the sense-making process. Journal of Research in Science Teaching, 37 (5), 449-457.

Shelley, C. (1999). Multiple analogies in evolutionary biology. Studies in History and Philosophy of Biology and Biomedical Sciences, 30(2), 143-180.

Short, T. (2002). Darwin's concept of final cause: Neither new nor trivial. Biology and Philosophy, 17(3), 322-340.

Smith, M. (2010). Current status of research in teaching and learning evolution: II. Pedagogical Issues. Science and Education, 19(4-8), 523-538.

Sober, E. (1996). Filosofía de la biología. Madrid, España: Alianza.

Solan Wilson, D. (2010), Evolución para todos. De cómo la teoría de la evolución cambia nuestro pensar. Xalapa, México: Universidad Veracruzana.

Sterelny, K. y Griffiths, P. (1999). Sex and Death. An introduction to philosophy of biology. Chicago: The University of Chicago Press.

Sterrett, S, (2002). Darwin's analogy between artificial and natural selection: How does it go? Studies in History and Philosophy of Biology and Biomedical Sciences, 33(1), 151-168.

Stryer, L. (1995). Bioquímica. Barcelona: Reverté.

Tamir, P. y Zohar, A. (1991). Anthropomorphism and teleology in reasoning about biological phenomena. Science Education, 75(1), 57-67. 
Thagard, P. (1995). Mental leaps: Analogy in creative thought. Cambridge, Inglaterra: MIT Press.

Theunissen, B. (2012). Darwin and his pigeons. The analogy between artificial and natural selection revisited. Journal of the History of Biology, 45(2), 179-212.

Villamar, A.; Noguera Solano, R. y Ruiz Gutiérrez, R. (2007). Metáforas e interdisciplina en la teoría darwiniana. En J. Muñoz Rubio, (coord.) La interdisciplina y las grandes teorías del mundo moderno. México D. F.: Universidad Nacional Autónoma de México.

Walsh, D. (2000). Chasing shadows: Natural selection and adaptation. Studies in History and Philosophy of Sciences, 37 (1), 135-153.

Wilner, E. (2006). Darwin's artificial selection as an experiment. Studies in History and Philosophy of Biology and Biomedical Sciences, 37(1), 26-40.

Zohar, A. y Ginossar, S. (1998). Lifting the taboo regarding teleology and anthropomorphism in biology. Education-Heretical Suggestions. Science Education, 82(6), 679-697.

Zohar, A. y Yehudit Jody, D. (eds.). (2012). Metacognition in Science Education. Trends in Current Research. Dordretch, Holanda: Springer. 